\title{
Determining the Validity And Reproducibility of A Feeding Assessment Tool to Assess Nutrient Intake in New Zealand Infants Aged 9 to 12 Months ${ }^{+}$
}

\author{
Amy Judd 1,*, Kathryn Beck ${ }^{2}$, Chris McKinlay ${ }^{3}$ and Cathryn Conlon ${ }^{2}$ \\ 1 School of Sport, Exercise and Nutrition, Massey University, Auckland 0632, New Zealand \\ 2 School of Sport, Exercise and Nutrition, College of Health, Massey University, Auckland 0632, \\ New Zealand; K.L.Beck@massey.ac.nz (K.B.); c.conlon@massey.ac.nz (C.C.) \\ 3 Liggins Institute and Department of Paediatrics, Child and Youth Health, University of Auckland, \\ Auckland 1023, New Zealand; c.mckinlay@auckland.ac.nz \\ * Correspondence: rusamy@gmail.com \\ + Presented at the 2018 Nutrition Society of New Zealand Annual Conference, Auckland, New Zealand, \\ 28-30 November 2018.
}

Published: 14 March 2019

Background: Dietary assessment in infants is challenging but necessary to understand the relationship between nutrition and growth and development. However there are currently no simple, validated dietary assessment methods that assess nutrient intake in New Zealand infants. Our objective was to assess the relative validity and reproducibility of a complementary feeding questionnaire (CFQ) to determine nutrient intakes of New Zealand infants aged 9-12 months.

Methods: A cross-sectional study design was used. Ninety-five parent-infant pairs (infant age $10 \pm 1$ months) completed the CFQ twice (CFQ-1 and CFQ-2), approximately 4 weeks apart. A fourday weighed food record (4dWFR) was collected on non-consecutive days between CFQ administrations. Validity and reproducibility were assessed for intakes of energy and 18 nutrients using paired $t$-tests, Pearson's correlation coefficients, cross-classification and Bland-Altman analysis.

Results: Most nutrient intakes from the CFQ were comparable to the $4 \mathrm{dWFR}$ (range $<1 \%$ to $28.2 \%$ different). Correlation coefficients ranged from $r=0.18$ (saturated fat) to $r=0.81$ (iron; mean $r=0.52$ ). The majority of nutrients showed good cross-classification $(>50 \%$ correctly classified and $<10 \%$ grossly misclassified). Over half (53.9\%) of participants were correctly classified by both methods (range $39.0 \%$ to $67.4 \%$ ). Between $2.1 \%$ and $14.7 \%$ of participants (mean $7.1 \%$ ) were grossly misclassified. Most nutrients showed acceptable agreement between methods $(\kappa=0.20-0.60)$. For reproducibility, all nutrients showed acceptable to good correlations $(r \geq 0.20)$, good crossclassification apart from fat and saturated fat $(40.9 \%$ and $47.3 \%$, respectively) and all had less than $10 \%$ grossly misclassified. All nutrients showed acceptable to good agreement $(\kappa>0.20)$.

Conclusions: The CFQ appears to have acceptable validity and good reproducibility for assessing nutrient intake in infants aged 9-12 months, making it a useful tool for use in future research.

Supplementary Material: The poster is available online atwww.mdpi.com/2504-3900/8/1/53/s1.

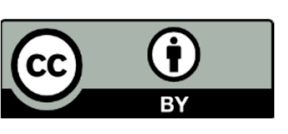

(C) 2019 by the authors. Licensee MDPI, Basel, Switzerland. This article is an open access article distributed under the terms and conditions of the Creative Commons Attribution (CC BY) license (http://creativecommons.org/licenses/by/4.0/). 